Draft VERSion MAY 15, 2020

Typeset using $\mathrm{LAT}_{\mathrm{E}} \mathrm{X}$ twocolumn style in AASTeX63

\title{
Search for Continuous Gravitational Waves from the Central Compact Objects in Supernova Remnants Cassiopeia A, Vela Jr. and G347.3-0.5
}

\author{
M. Alessandra Papa,${ }^{1,2,3}$ J. Ming, ${ }^{1,3}$ E.V. Gotthelf,${ }^{4}$ B. Allen,,${ }^{1,2,3}$ R. Prix,,${ }^{1,3}$ V. Dergachev ${ }^{1,3}$ \\ H.-B. Eggenstein, ${ }^{1,3}$ A. Singh, ${ }^{1,3,5}$ And S. J. Zhu ${ }^{1,6}$ \\ ${ }^{1}$ Max Planck Institute for Gravitational Physics (Albert Einstein Institute), Callinstrasse 38, 30167 Hannover, Germany \\ ${ }^{2}$ University of Wisconsin Milwaukee, 3135 N Maryland Ave, Milwaukee, WI 53211, USA \\ ${ }^{3}$ Leibniz Universität Hannover, D-30167 Hannover, Germany \\ ${ }^{4}$ Columbia Astrophysics Laboratory, Columbia University, 550West 120th Street, New York, NY 10027-6601, USA \\ ${ }^{5}$ The Geophysical Institute, Bjerknes Centre for Climate Research, University of Bergen, 5007 Bergen, Norway \\ ${ }^{6}$ DESY, 15738 Zeuthen, Germany
}

(Accepted May 11, 2020)

Submitted to ApJ

\begin{abstract}
We perform a sub-threshold follow-up search for continuous nearly-monochromatic gravitational waves from the central compact objects associated with the supernova remnants Vela Jr., Cassiopeia A, and SNR G347.3-0.5. Across the three targets, we investigate the most promising $\approx 10,000$ combinations of gravitational wave frequency and frequency derivative values, based on the results from an Einstein@Home search of the LIGO O1 observing run data, dedicated to these objects. The selection threshold is set so that a signal could be confirmed using the newly released O2 run LIGO data. In order to achieve best sensitivity we perform two separate follow-up searches, on two distinct stretches of the $\mathrm{O} 2$ data. Only one candidate survives the first $\mathrm{O} 2$ follow-up investigation, associated with the central compact object in SNR G347.3-0.5, but it is not conclusively confirmed. In order to assess a possible astrophysical origin we use archival X-ray observations and search for amplitude modulations of a pulsed signal at the putative rotation frequency of the neutron star and its harmonics. This is the first extensive electromagnetic follow-up of a continuous gravitational wave candidate performed to date. No significant associated signal is identified. New X-ray observations contemporaneous with the LIGO O3 run will enable a more sensitive search for an electromagnetic counterpart. A focused gravitational wave search in $\mathrm{O} 3$ data based on the parameters provided here should be easily able to shed light on the nature of this outlier. Noise investigations on the LIGO instruments could also reveal the presence of a coherent contamination.
\end{abstract}

Keywords: gravitational waves — continuous - SNRs - pulsar - J232327.9+584842 — CXOU J085201.4-461753 — 1WGA J1713.4-3949

\section{INTRODUCTION}

Corresponding author: M.Alessandra Papa

maria.alessandra.papa@aei.mpg.de

jing.ming@aei.mpg.de

eric@astro.columbia.edu
At the time of writing, gravitational waves from 14 binary black hole mergers and a binary neutron star merger have been reported (Abbott et al. 2020; Venumadhav et al. 2020; Nitz et al. 2019; Abbott et al. 2019a). All of these signals are visible in the detectors for less than a second, or several tens of seconds for a binary neutron star merger, and present a strain amplitude of $\sim 10^{-21}$ for most of that time. 
This paper is about a different type of signal: a persistent train of nearly monochromatic gravitational waves with an amplitude four (or more) orders of magnitude weaker than that of the binary merger signals.

Various physical mechanisms have been suggested that could give rise to continuous gravitational waves: deformations of the compact object, including asymmetric mass distribution (Johnson-McDaniel \& Owen 2013), ringing (r-modes) (Owen et al. 1998), and non axi-symmetric flows of bulk matter induced by differential rotation between the core of the star and its crust through Ekman pumping (Singh 2017), as well as more exotic scenarios, for instance involving very low mass cold dark matter binaries (Horowitz \& Reddy 2019; Horowitz et al. 2020) or emission from boson clouds around black holes (Arvanitaki et al. 2015; Zhu et al. 2020).

The relationship between the spin frequency of the star and the frequency of the continuous gravitational wave signal depends on the emission mechanism but typically the continuous gravitational wave frequency does not exceed twice the spin frequency. So, in the high sensitive band of the LIGO detectors - between $20 \mathrm{~Hz}$ and $2 \mathrm{kHz}$ - we expect continuous gravitational waves from neutron stars with spin periods in the range 1-100 ms.

Perhaps among the most promising candidates for continuous gravitational wave emission are young, isolated neutron stars. These could have a high spindown and hence a potentially large energy budget to be radiated away in gravitational waves.

The central compact objects associated with supernova remnants could indeed be young isolated neutron stars. We know of $\sim 10$ objects at the center of supernova remnants (Gotthelf et al. 2013). Three of these objects are known pulsars (with spin periods $P=105,112,424 \mathrm{~ms}$ ) whose slow spin-down rate implies a low spin-down power and weak magnetic dipole field. The rest are undetected as pulsars and their timing properties remain a mystery.

In the past the youngest/closest supernova remnants with identified central compact objects have been chosen as targets for gravitational wave searches that span a broad range of physically plausible signal frequencies and frequency-derivatives (Lindblom \& Owen 2020; Millhouse et al. 2020; Ming et al. 2019; Abbott et al. 2019b; Zhu et al. 2016; Aasi et al. 2015a).

In this paper we use the released LIGO O2 data set to further the results of the O1-data search (Ming et al. 2019) for continuous gravitational wave emission from the compact object in the supernova remnants Vela Jr., Cassiopeia A and SNR G347.3-0.5. The targets and search setups were chosen based on an optimization scheme that maximizes the total detection probability over search setups and astrophysical targets, assuming a total computing budget of a few months on the Einstein@Home volunteer distributed computing project (Einstein@Home 2019; Ming et al. 2016, 2018). The detection probability depends on the assumed source and signal properties (distance, age, ellipticity, frequency) as well as on the sensitivity of the search pipeline (search setup and search parameters, noise level).

In Section 2.1, we present the results of the subthreshold search of Einstein@Home candidates from Ming et al. (2019). In Section 2.2 we describe the followup search using part of the LIGO O2 data set. With this search we find a marginal candidate for the source associated with SNR G347.3-0.5, which is however not definitely confirmed in the second part of the O2 data (Section 2.3.1). We report a search for an electromagnetic counterpart for this candidate in Section 2.3.2. In Section 3 we compute the upper limits on the intrinsic gravitational wave amplitude stemming from the LIGO search. Finally, in Section 4, we summarize our conclusions.

\section{THE SEARCH}

The search described in this paper targets nearly monochromatic gravitational waves (see for example Section II of Jaranowski et al. (1998)) from compact objects in three supernova remnants, CXOU J232327.9+584842 in Cassiopeia A (Tananbaum 1999), CXOU J085201.4-461753 in Vela Jr. (Slane et al. 2001), and 1WGA J1713.4-3949 in SNR G347.3-0.5 (Pferffermann \& Aschenbach 1996), referred to herein as J232327, J085201, and J1713, respectively. No pulsations have been reported from any of these sources.

We use public data from the first (O1; 2015-2016) and second (O2; 2017) run of the advanced LIGO detectors (Abbott et al. 2016; Aasi et al. 2015b; LIGO 2018, 2019; Vallisneri et al. 2015). Measured on a fully coherent search, and assuming that the $\mathrm{O} 1$ and $\mathrm{O} 2$ sensitivities are comparable, the relative strain sensitivity improvement from using the $\mathrm{O} 2$ data over the $\mathrm{O} 1$ data is $\sim 27 \%$. But the real value of the $\mathrm{O} 2$ data for follow-ups of candidates, is not in the raw sensitivity gain. It rather lies in that the new data is independent of the data where the candidates are originally identified. Together with the fact that the follow-up searches cover a more limited parameter space than the original ones, this makes it possible to assess the significance of a finding on the new data, including confidently claiming a detection.

We use a large-scale hierarchical scheme that was first deployed in Papa et al. (2016) and that has now become standard practice in broad continuous wave searches 
Table 1. Search ranges. The spindown ranges quoted are the ones used at $f=100 \mathrm{~Hz}$. The ranges at different frequencies are readily derived from Eq. 1. The barycentric reference time (the epoch of the ephemeris) is $T_{\text {ref }}=1131943508$ GPS s corresponding to MJD 57345.1986016667 (TDB).

\begin{tabular}{lccc}
\hline \hline COMPACT OBJECT & J232327 & J085201 & J1713 \\
REMNANT & $($ G111.7-02.1 or Cas A) & $($ G266.2-1.2 or Vela Jr.) & $($ G347.3-0.5) \\
references & (Tananbaum 1999) & (Pavlov et al. 2001) & (Mignani et al. 2008) \\
\hline$f$ range & {$[20-1500] \mathrm{Hz}$} & {$[20-1500] \mathrm{Hz}$} & {$[20-1500] \mathrm{Hz}$} \\
$\dot{f}$ range (@ 100 Hz) & {$\left[-9.6 \times 10^{-9}-0\right] \mathrm{Hz} / \mathrm{s}$} & {$\left[-4.5 \times 10^{-9}-0\right] \mathrm{Hz} / \mathrm{s}$} & {$\left[-2.0 \times 10^{-9}-0\right] \mathrm{Hz} / \mathrm{s}$} \\
$\ddot{f}$ range (@ 100 Hz) & {$\left[0-4.6 \times 10^{-18}\right] \mathrm{Hz} / \mathrm{s}^{2}$} & {$\left[0-1.0 \times 10^{-18}\right] \mathrm{Hz} / \mathrm{s}^{2}$} & {$\left[0-2.0 \times 10^{-19}\right] \mathrm{Hz} / \mathrm{s}^{2}$} \\
RA (J2000, in radians) & 6.123770 & 2.3213891 & 4.5093705 \\
DEC (J2000, in radians) & 1.026458 & -0.8080543 & -0.6951891 \\
\hline
\end{tabular}

Table 2. Spacings on the signal parameters used for the templates in the Einstein@Home search.

\begin{tabular}{cccc}
\hline \hline $\begin{array}{c}\text { COMPACT OBJECT } \\
\text { REMNANT }\end{array}$ & G111.7-02.1 or Cas A & G266.2-1.2 or Vela Jr. & G347.3-0.5 \\
\hline$T_{\text {coh }}$ & $245 \mathrm{hr}$ & $369 \mathrm{hr}$ & $489 \mathrm{hr}$ \\
\hline$N_{\text {seg }}$ & 12 & 8 & 6 \\
$\delta f$ & $6.85 \times 10^{-7} \mathrm{~Hz}$ & $3.21 \times 10^{-7} \mathrm{~Hz}$ & $2.43 \times 10^{-7} \mathrm{~Hz}$ \\
$\delta \dot{f}_{c}$ & $3.88 \times 10^{-12} \mathrm{~Hz} / \mathrm{s}$ & $1.33 \times 10^{-12} \mathrm{~Hz} / \mathrm{s}$ & $6.16 \times 10^{-13} \mathrm{~Hz} / \mathrm{s}$ \\
$\gamma_{1}$ & 5 & 9 & 9 \\
$\delta \ddot{f}_{c}$ & $4.03 \times 10^{-18} \mathrm{~Hz} / \mathrm{s}^{2}$ & $1.18 \times 10^{-18} \mathrm{~Hz} / \mathrm{s}^{2}$ & $5.07 \times 10^{-19} \mathrm{~Hz} / \mathrm{s}^{2}$ \\
$\gamma_{2}$ & 21 & 21 & 11 \\
\hline
\end{tabular}

(Abbott et al. 2017; V. Dergachev \& Eggenstein 2019; Abbott et al. 2019c; Dergachev \& Papa 2019; Palomba et al. 2019; Dergachev \& Papa 2020). The first, and most computationally intensive search on the $\mathrm{O} 1$ data is carried out on the Einstein@Home volunteer computing project and it is followed by two follow-up stages on candidates above threshold. We refer to these as Stage-0, Stage-1 and Stage-2. The setup of the Einstein@Home search and of the $\mathrm{O} 1$ follow-up stages is described in Ming et al. (2019). The thresholds used here are lower than the ones used in Ming et al. (2019) and candidates exist that survive all O1 follow-up stages. These surviving candidates are further inspected using $\mathrm{O} 2$ data.

\subsection{The 01 searches}

The searched waveforms are defined by frequency $f$ and frequency derivative parameters $\dot{f}$ and $\ddot{f}$ in these ranges:

$$
\left\{\begin{array}{l}
-f / \tau \leq \dot{f} \leq 0 \mathrm{~Hz} / \mathrm{s} \\
0 \mathrm{~Hz} / \mathrm{s}^{2} \leq \ddot{f} \leq 5|\dot{f}|_{\max }^{2} / f=5 f / \tau^{2} .
\end{array}\right.
$$

The ages $\tau$ of the compact objects are taken equal to 330 yrs, 700 yrs, 1600 yrs for J232327, J085201, and J1713 respectively. These age values incorporate the uncertainties on the birth-dates of our targets (Wang et al. 1997; Iyudin 1999; Fesen et al. 2006; Allen et al. 2015), airing towards the smaller estimates, which yield a broader (and hence more conservative, safer) search range - see Ming et al. (2018) for more details. The frequency derivative ranges for $f=100 \mathrm{~Hz}$ are shown in Table 1, for reference. The frequency and frequency derivatives are defined at the barycentric reference time (epoch of the ephemeris) $T_{\text {ref }}$, which is first given in Table 1 . 
The basic building block of the hierarchical search is the stack-slide semi-coherent search that uses the global correlation transform (GCT) method (Pletsch \& Allen 2009; Pletsch 2008, 2010): The data is divided in segments, and each segment is searched coherently combining the data from both detectors with a matched filter called the $\mathcal{F}$-statisitic (Cutler \& Schutz 2005). The $\mathcal{F}$ statisitic optimally combines together the data from the two detectors at different times based on their noise level and the antenna sensitivity pattern and also references arrival time to the solar system barycenter based on the sub-arcsec coordinates for each target. The results from these coherent searches are appropriately summed, one per segment, yielding an average detection statistic value over all the segments, $\overline{\mathcal{F}}$. For a stack-slide search on Gaussian noise, $N_{\text {seg }} \times 2 \overline{\mathcal{F}}$ follows a chi-squared distribution with $4 N_{\text {seg }}$ degrees of freedom, $\chi_{4 N_{\text {seg }}}^{2}$. When $N_{\text {seg }}=1$ the search becomes a fully coherent search.

In Table 2 we show the main parameters of the Stage0 searches: the frequency spacing $\delta f$ and the first and second order frequency derivative spacings $\delta \dot{f}=\gamma_{1} \delta \dot{f}_{c}$ and $\delta \ddot{f}=\gamma_{2} \delta \ddot{f}_{c}$. We refer the reader to Ming et al. (2019) for a detailed description of the search setup.

Based on the coherent $\mathcal{F}$-statistics, a new statistics is constructed that is robust to the presence of persistent and transient lines in the data: $\hat{\beta}_{\mathrm{S} / \mathrm{GLtL}}$. This detection statistic tests the signal hypothesis not only against the Gaussian noise hypothesis but also against the hypothesis that a persistent or transient spectral artefact in one of the two data streams is contaminating the data (Keitel et al. 2014; Keitel 2016). $\hat{\beta}_{\mathrm{S} / \mathrm{GLtL}}$ eases the impact on the detection efficiency and false alarm rate of various types of spectral artefacts in the data and we use it to rank the search results on the volunteer computers and based on it, select the results that are returned to the main server.

A result, or candidate, is defined by the targeted sky position (one of three), by the frequency, first- and second-order frequency derivatives and by its detection statistic value.

We consider only the "undisturbed" frequency bands identified in Ming et al. (2019), i.e. frequency bands where the bulk of the search results do not display major deviations from what is expected in the absence of large noise artefacts. We apply to the candidates from these bands the same semi-coherent DM-off veto (S. J. Zhu \& Walsh 2017) and the same clustering procedure as in Ming et al. (2019); A. Singh \& Walsh (2017). The DM-off veto discards candidates whose detection statistic increases when the astrophysical Doppler modulation is removed from the searched signal waveform (Doppler Modulation-off). The clustering procedure bundles to- gether high detection statistic values which are close enough in parameter space that they can be ascribed to the same root cause. This saves computations in the follow-up stages because not all close-by candidates are searched independently.

From this set we further select those candidates whose detection statistic value lies above a given threshold. Since the number of templates covered in a fixed frequency interval increases with frequency, we set a different detection threshold in the frequency ranges [20-250] $\mathrm{Hz},[250-520] \mathrm{Hz}$ and [520-1500] Hz. We refer to these as the low, mid and high frequency range, respectively. Table 3 shows the values of the thresholds for the different targets and frequencies. The thresholds are such that overall a few thousand candidates are followed up from each target. We use different thresholds for the different targets in the same frequency range because a different number of waveforms are probed for the different targets, due to the different age: for the younger targets a broader range of frequency derivatives is sampled.

The Stage-1 and Stage-2 follow-ups use the same search setup for all targets, with a coherent timebaseline of about 60 days (1440 hrs) and the entire O1 data set, respectively. Candidates survive from one stage to the next if their detection statistic value increases consistently with what is expected from a signal.

Initially we have 3275 candidates from the J085201 search, 2269 from J232327 and 3918 from J1713. The DM-off veto rejects 557, 1223 and 1032 candidates for J085201, J232327 and J1713, respectively. Figure 1 shows the detection statistic values of these candidates as a function of frequency throughout these follow-up stages and Figure 2 the distribution of their detection statistic values.

After each follow-up search the number of surviving candidates decreases and at the end of the last stage we are left with 575 candidates of which 142 are from J085201, 81 from J232327 and 352 from J1713. We note that the fraction of candidates surviving the last stage on $\mathrm{O} 1$ data is about $4 \%$ of the original ones for J085201 and J232327 and 9\% for J1713. This is consistent with the initial search setup for J1713 having a significantly longer coherence time-baseline compared to the other searches, and hence the follow-up searches having less discriminatory power for the J1713 candidates, that were selected based on more stringent criteria, to start with.

\subsection{The follow-up searches on the first half of O2}

In order to reduce the trials factor of the last stage follow-up, we perform two separate searches in O2. For the first follow-up search on $\mathrm{O} 2$ data we only use a por- 


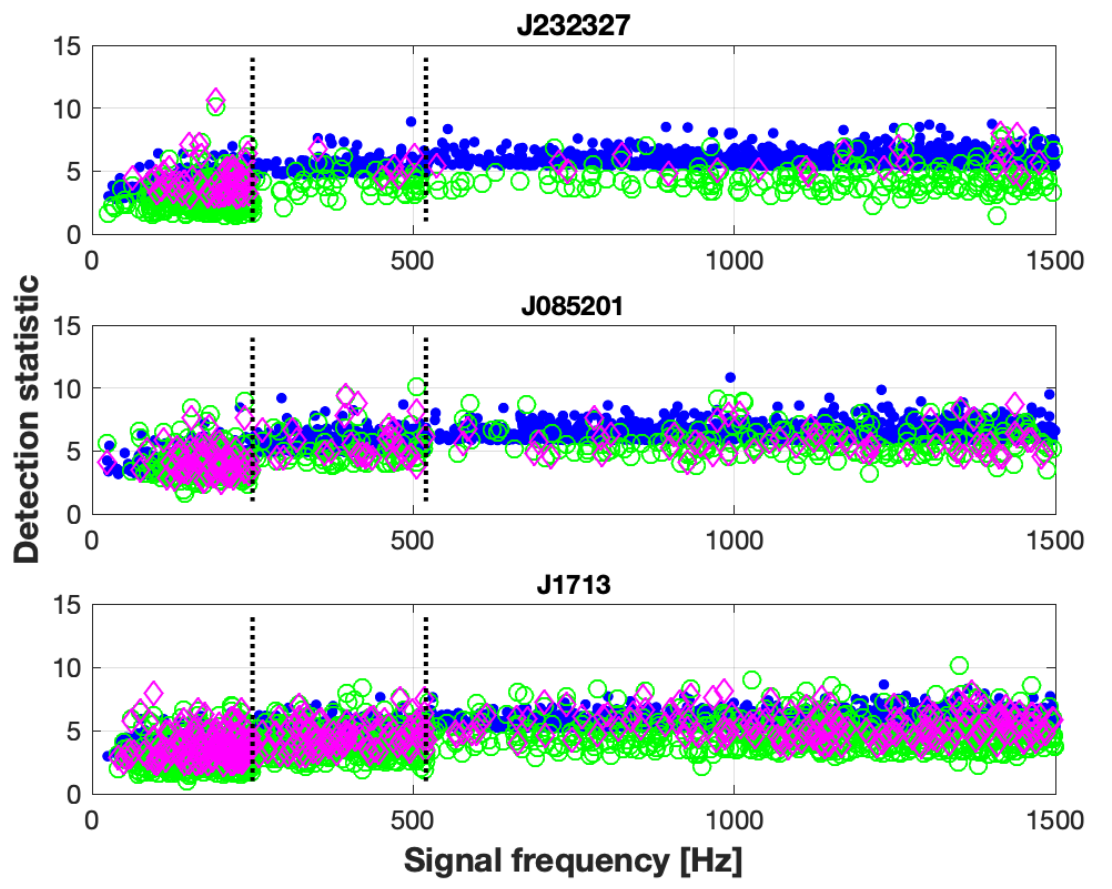

Figure 1. Detection statistic $\hat{\beta}_{\mathrm{S} / \mathrm{GLtL} r}$ as a function of signal frequency of candidates. The smaller filled circles (blue) are the candidates above the Stage-0 threshold that also passed the semi-coherent DM-off veto and are hence searched in Stage-1. The larger (green) circles indicate those candidates that survive Stage- 1 and are searched with Stage-2, the fully coherent search using O1 data. The diamonds (magenta) show the candidates that survive Stage-2. The dotted vertical lines indicate the boundaries of the different frequency ranges for the outlier selection (see Table 3). The maximum value of the detection statistics increases with frequency because the number of searched templates increases with frequency. A J232327 candidate with detection statistic $\sim 10$ catches the eye, which however is not confirmed in the first follow-up on O2 data (see the middle panel of Figure 3), which means that whatever in the data matched a signal waveform in $\mathrm{O} 1$ data yielding that outlier was not there any more in the first half of the $\mathrm{O} 2$ data $(\mathrm{O} 2 \mathrm{a})$.
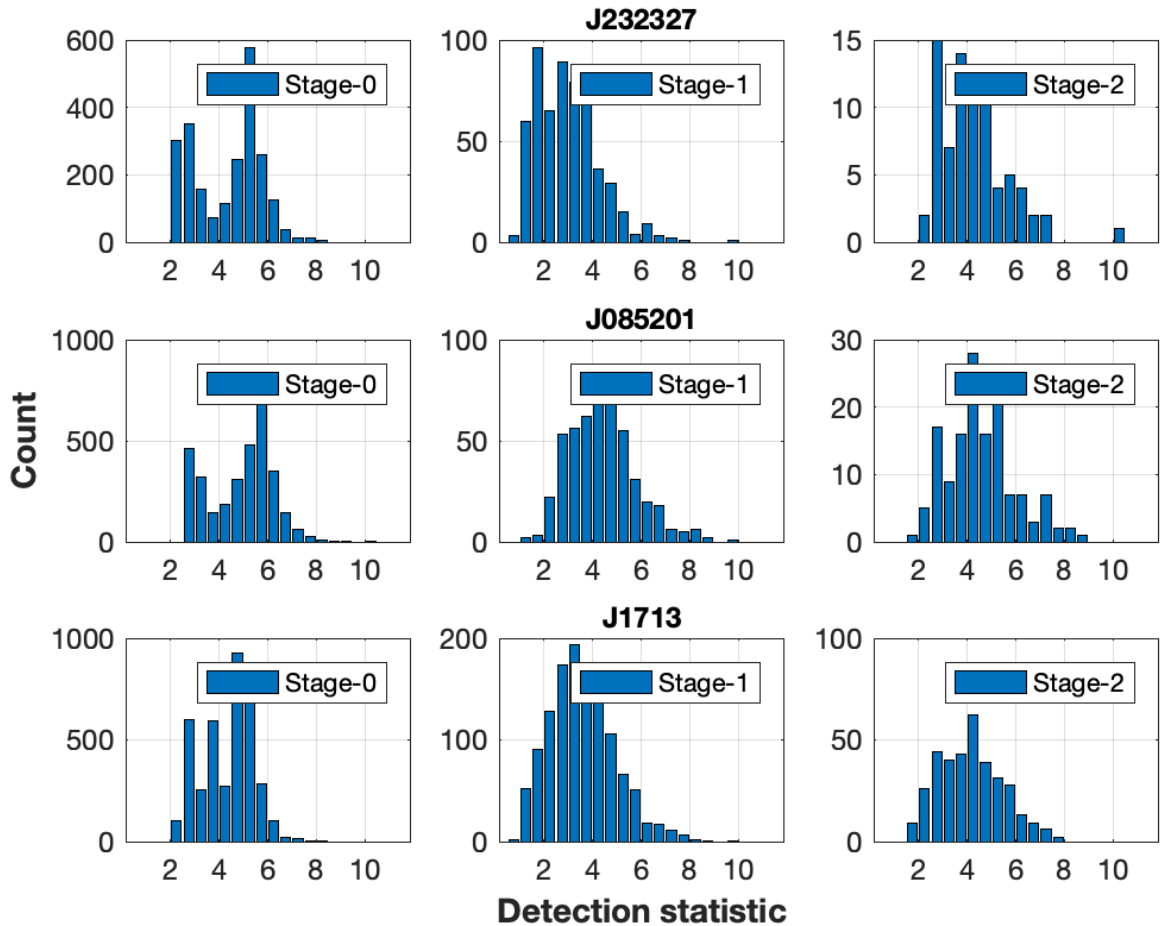

Figure 2. The distributions of the detection statistic $\hat{\beta}_{\mathrm{S} / \mathrm{GLtL} r}$ of candidates that survive each of the three O1 stages, across the entire frequency band. The J1713 candidate at $\approx 369 \mathrm{~Hz}$ discussed in Section 2.3 has $\hat{\beta}_{\mathrm{S} / \mathrm{GLtL} r} \approx 4$ throughout these stages. At this stage the candidate does not stand out among the other outliers under investigation but we point it out in light of the results of the O2a data follow-up. 
Table 3. Stage-0 detection statistic thresholds.

\begin{tabular}{cccc}
\hline \hline & J232327 & J085201 & J1713 \\
\hline$\hat{\beta}_{\mathrm{S} / \text { GLtL } r}^{\text {low-freq }}$ & 2.75 & 3.10 & 2.95 \\
$\hat{\beta}_{\mathrm{S} / \text { GLtL } r}^{\text {mid-freq }}$ & 4.75 & 4.9 & 4.05 \\
$\hat{\beta}_{\mathrm{S} \text { /GLtLr }}^{\text {high-freq }}$ & 5.42 & 5.86 & 5.02 \\
\hline
\end{tabular}

tion of the available data, O2a, which is comparable in duration with the $\mathrm{O} 1$ set and spans about 120 days. In fact, an interruption in the science run of LIGO between May 9th and June 8th 2017, provides a natural split of the data in two sets. We use the same search setup as for the last stage on $\mathrm{O} 1$, which is fully coherent. We search a region which corresponds to the $99 \%$ confidence signal-parameters containment region, based on signal simulation-and-recovery Monte Carlos. We consider the most significant result from each follow-up.

We follow-up only the outliers that do not fall in $\mathrm{O} 2$ spectral regions possibly contaminated by lines: 137 from J085201, 78 from J232327 and 331 from J1713, which amounts to $\sim 95 \%$ of the O1 outliers. Each search covers about 14 million templates. If these templates had no overlap, the 14 million results would be independent of each other and the distribution of the maximum over every 14 million template search for Gaussian noise would be known. In reality nearby templates present a large overlap: the grids are constructed in this way in order to reduce the false dismissal rate for signals whose parameters lie in-between the grid points. We find that the actual distribution for Gaussian noise data is well modelled assuming a smaller number of independent searches (8.2 million). This is what the solid line (red) in Figure 3 shows. The fit was performed on the results of identical searches on fake Gaussian noise, which is what the yellow bars show.

The darker (purple) bars in Figure 3 are the normalised histogram for the detection statistic of the results of the searches. For J232327 and J085201 the distributions are completely consistent with Gaussian noise (the lighter, yellow bars). For J1713 an outlier is noticeable at $2 \mathcal{F}_{r} \sim 56$.

The p-value associated with the J1713 most significant candidate is about $7.6 \times 10^{-5}$. This is an estimate of the probability that noise alone would produce a value of the detection statistic like the one associated with that candidate in a single follow-up search. Since 546 such searches were performed, this translates into an overall p-value of about $4 \%$. This is a marginal candidate but
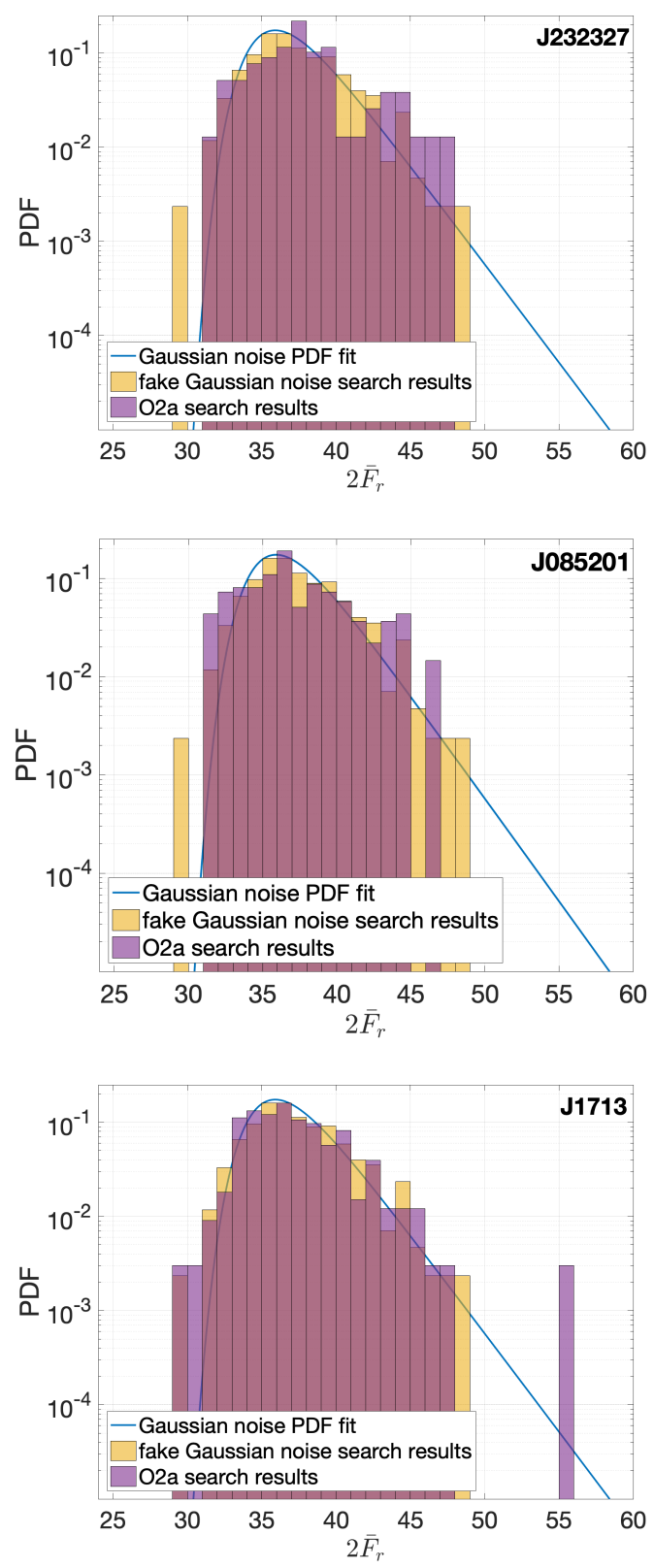

Figure 3. Distribution of the detection statistic $2 \overline{\mathcal{F}}_{r}$ from the O2a searches on each of the 137 candidates from J085201, 78 candidates from J232327 and 331 candidates from J1713. The subscript " $r$ " in $2 \overline{\mathcal{F}}_{r}$ refers to the detection statistic being recalculated exactly at the template points. This is a technical detail that we give here for completeness and to explain the subscript.

we have more data (the second half of O2) that can be used to validate it or discard it. We report further analyses on this candidate in the next section.

The O2a data set comprises $86 \%$ of the amount of data of the $\mathrm{O} 1$ data set, hence the detection statistic values from $\mathrm{O} 1$ and $\mathrm{O} 2 \mathrm{a}$ are remarkably consistent under the assumption of the $\mathrm{O} 1$ result being drawn from 
a distribution with a non-centrality parameter $\left(\mathrm{SNR}^{2}\right)$ $\rho^{2}=67.0-4$ and the O2a result being drawn from a distribution with a non centrality parameter $54=86 \%$ $\rho^{2}$.

\subsection{The J1713 candidate}

\subsubsection{The follow-up search on the second part of O2}

We perform a fully coherent search on the second "half" of $\mathrm{O} 2(\mathrm{O} 2 \mathrm{~b})$. The amount of data in this set is about $76 \%$ of the $\mathrm{O} 1$ data set and $89 \%$ of the $\mathrm{O} 2 \mathrm{a}$ data. The $2 \mathcal{F}$ expected in $\mathrm{O} 2 \mathrm{~b}$ based on $\mathrm{O} 1(\mathrm{O} 2 \mathrm{a})$ is 52.8 (50.1) with a standard deviation of at least $14^{1}$. The expected value for the $\mathrm{O} 2 \mathrm{~b}$ search due to Gaussian noise (based on Monte Carlos) is less than 32.3 with a standard deviation of $\sim 2.7$. The measured value of 34.7 has a Gaussian p-value of $10 \%$ and is within a 1.3 standard deviations of the expected value on the signal distribution.

We combine all the data and search the $\mathrm{O} 1+\mathrm{O} 2$ data set. It would be very demanding to carry out this search many times on Gaussian noise in order to provide an estimate of the $\mathrm{p}$-value as done for the $\mathrm{O} 2 \mathrm{~b}$ search. We hence resort to a conservative estimate of the Gaussiannoise significance based on the number of searched templates. The search covers $2.9 \times 10^{10}$ templates and if they were all independent the maximum expected value for $2 \mathcal{F}$ in Gaussian noise would be $\sim 56$ with a standard deviation of 2.7. The value that we find is 76.0. Based on the $\mathrm{O} 1$ value, the expected $\mathrm{O} 1+\mathrm{O} 22 \mathcal{F}$ is about 171.8 , with a standard deviation of at least 26 . The value that we find does not seem consistent with Gaussian noise but also falls short of the expectations based on the $\mathrm{O} 1$ signal-hypothesis by a few standard deviations.

A summary of the J1713 follow-up results is given in Table 4.

\subsubsection{X-ray data search}

As the central compact objects associated with the targeted SNRs have only been detected in the X-ray energy band, we use archival X-ray observations of 1WGA J1713.4-3949 acquired with sufficient time resolution to cross-check for an electromagnetic counterpart to the gravitational wave candidate. Table 5 presents the ROSAT, Rossi-XTE, and XMM-Newton observations used in this work. These observations are processed, reduced, and analyzed following the standard procedures for each mission. The photon arrival times are corrected

1 This holds under the assumption that the non-centrality parameter estimate carries no uncertainty. This is not the case, so these numbers are a lower estimate of the standard deviation, which can actually be larger by up to a factor of 2 (Prix 2017). to the solar system barycenter using the Chandra coordinates of J1713 (Mignani et al. 2008).

For the ROSAT data, source photons are extracted from a $r<0.2^{\prime}$ circular aperture yielding 1343 counts for the $31 \mathrm{ks}$ exposure in the $0.1-2.5 \mathrm{keV}$ ROSAT bandpass. The XMM-Newton EPIC-MOS data are obtained in one-dimensional continuous clocking mode (CCmode) with $1.5 \mathrm{~ms}$ timing resolution, corresponding to a Nyquist frequency of $333.3 \mathrm{~Hz}$. Data are extracted from the central $0.6^{\prime}$ wide region of the CCD for a total of 59,781 source counts in the $0.5-5 \mathrm{keV}$ optimal range for the central compact object. The Rossi-XTE data is restricted to the top layer of the proportional counter $(2-5 \mathrm{keV})$ whose lower limit just covers the harder end of the soft central compact object spectrum $(\sim 0.5-5 \mathrm{keV})$, resulting in reduced sensitivity for the pulsar search using a total of 87,305 counts.

We search these data sets for a coherent pulsed signal over the full Nyquist range including the three basic electromagnetic rotation frequency $f_{r}$ ranges compatible with the gravitational wave frequency $f$, namely at $f_{r}=$ $f, f_{r}=f / 2$, and the broad r-mode interval $f_{r} \approx 3 / 4 f$. We first look for a strong signal within the full range of possible frequencies, namely, $100<f_{r}<400 \mathrm{~Hz}^{2}$, that allows for the large time span between the X-ray and LIGO data sets, and the extrapolated uncertainties in the spin-down measurement, conservatively estimated as $\delta f=5 \times 10^{-5} \mathrm{~Hz}$ and $\delta \dot{f}=0.002 \times 10^{-9} \mathrm{~Hz} / \mathrm{s}$. We also conduct a more sensitive narrow range search around the candidate parameters $(f, \dot{f})$ extrapolated to the X-ray data epoch, also allowing for the uncertainties quoted above.

We use the $\mathrm{O} 1+\mathrm{O} 2$ search parameters given in the last column of Table 4. The rapid spin-down associated with this signal requires that we include the first derivative in the search. We evaluate the signal significance using an FFT algorithm, the $Z_{n}^{2}$ test (Buccheri et al. 1983), where $\mathrm{n}=1,2,3$ harmonics, and the H-test (de Jager et al. 1989), to check for power at higher harmonics. In these searches, we oversample the parameter space by a factor of 2 .

Given the large time span of the data sets and the small number of photons, it is most practical to perform the timing search using GPU technology applied to discrete Fourier transform algorithms $\left(Z_{n}^{2}\right.$, H-test) instead of FFTs. In the case of $X M M$-Newton, the upper range of the search is limited by the Nyquist frequency for this data set. For the Rossi-XTE data, because of the

\footnotetext{
${ }^{2}$ With the exception of the XMM-Newton data set for which $100<$ $f_{r}<333.3 \mathrm{~Hz}$.
} 
Table 4. Parameters of the most significant results from the high-resolution follow-ups around the most significant candidate from this search, which comes from J1713. The barycentric reference time (the epoch of the ephemeris) is $T_{\text {ref }}=1131943508$ GPS s, corresponding to MJD 57345.1986016667 (TDB).

\begin{tabular}{|c|c|c|c|c|c|}
\hline & O1 & $\mathrm{O} 2 \mathrm{a}$ & $\mathrm{O} 2 \mathrm{~b}$ & $\mathrm{O} 2$ & $\mathrm{O} 1+\mathrm{O} 2$ \\
\hline & Sept 2015 - Jan 2016 & Jan 2017 - May 2017 & Jun 2017 - Aug 2017 & Jan 2017 - Aug 2017 & Sept 2015 - Aug 2017 \\
\hline$f[\mathrm{~Hz}]$ & 368.8013845 & 368.8013794 & 368.8014296 & 368.8013795 & 368.8013845 \\
\hline$\dot{f}[\mathrm{~Hz} / \mathrm{s}]$ & $-4.378054 \times 10^{-9}$ & $-4.37800 \times 10^{-9}$ & $-4.37800 \times 10^{-9}$ & $-4.37800 \times 10^{-9}$ & $-4.37805 \times 10^{-9}$ \\
\hline$\ddot{f}\left[\mathrm{~Hz} / \mathrm{s}^{2}\right]$ & $5.7 \times 10^{-19}$ & $5.9 \times 10^{-19}$ & $5.5 \times 10^{-19}$ & $5.9 \times 10^{-19}$ & $5.7 \times 10^{-19}$ \\
\hline $2 \mathcal{F}$ & 67.0 & 57.0 & 34.7 & 53.3 & 76.0 \\
\hline$\propto \mathrm{SNR}^{2} / \mathrm{T}_{\text {data }}\left[\mathrm{s}^{-1}\right]$ & 1 & 0.98 & 0.64 & 0.48 & 0.44 \\
\hline
\end{tabular}

Table 5. Logs of the X-ray observations of RX J1713.4-3949 used in our search for an electromagnetic counterpart to the J1713 gravitational wave outlier.

\begin{tabular}{cccccccc}
\hline \hline \multirow{2}{*}{ ObsID } & $\begin{array}{c}\text { Mission/ } \\
\text { Instr }\end{array}$ & Data Mode & Start Date & $\begin{array}{c}\text { Exposure } \\
\text { UTC }\end{array}$ & $\begin{array}{c}\text { Span } \\
(\mathrm{ks})\end{array}$ & $\begin{array}{c}\text { Energy } \\
(\mathrm{ks})\end{array}$ & $\begin{array}{c}\text { Res. } \\
(\mathrm{keV})\end{array}$ \\
\hline 500466 & ROSAT/HRI & Nominal & $1996-08-31$ & 31 & 901 & $0.1-2.5$ & 61 \\
$40145-01$ & RXTE/PCA & GoodXenon & $1999-06-14$ & 8.4 & 905 & $2-5$ & 1 \\
0722190101 & XMM/MOS & CC-mode & $2013-08-24$ & 139 & 139 & $0.5-5$ & 1500 \\
\hline
\end{tabular}

large span between closely grouped data segments, in order to evaluate the power as a function of frequency, we average fixed sized FFTs on 5 available data intervals corresponding to these groups.

None of the above searches of the X-ray data yields a significant $(>4 \sigma)$ signal close to the expected X-ray frequencies $f_{r}$ and $2 f_{r}$, or ones consistent with the rmode range.

\section{UPPER LIMITS}

We set frequentist $90 \%$ confidence upper limits on the maximum gravitational wave amplitude consistent with our results as function of the signal frequency, $h_{0}^{90 \%}(f)$. Specifically, $h_{0}^{90 \%}(f)$ is the expected gravitational-wave intrinsic amplitude such that $90 \%$ of a population of signals with parameter values in our search range would have been detected by our search. In the absence of a detection these represent the smallest amplitude signals that we can exclude. The $h_{0}^{90 \%}(f)$ are determined through search-and-recovery Monte Carlos aimed at measuring the detection efficiency on a set of simulated signals added to the real data. More details of the standard procedure are provided in Ming et al. (2019). The basic idea is that the confidence level $-90 \%$ in this case - is the detection efficiency measured through the
Monte Carlos and the $h_{0}^{90 \%}(f)$ corresponds to the signal amplitude such that the detection confidence is $90 \%$. The most constraining upper limits are all close to signal frequencies of $\sim 172.5 \mathrm{~Hz}$ and are $1.2 \times 10^{-25}$ for CasA, $9.3 \times 10^{-26}$ for J085201 and $8.8 \times 10^{-26}$ for J1713. Figures 4 show all the upper limit curves discussed in this section.

The sensitivity depth $\mathcal{D}^{90 \%}$ (Behnke et al. 2015; Dreissigacker et al. 2018) expresses the sensitivity of a search:

$$
\mathcal{D}^{90 \%}:=\frac{\sqrt{S_{h}(f)}}{h_{0}^{90 \%}(f)}[1 / \sqrt{\mathrm{Hz}}]
$$

where $\sqrt{S_{h}(f)}$ is the noise level associated with a signal of frequency $f$, it is the same as the one reported in Ming et al. (2019) and it is available in machine-readable format at AEI (2020). Table 6 shows the sensitivity depth values of the searches in the different frequency ranges. As expected, the sensitivity depth is larger for the targets searched with an initial longer coherent baseline, i.e. for the older targets (J1713 is the eldest and then comes J085201). Furthermore, as the number of spindown templates increases, due to the trials factor, the detection threshold is increased (see Table 3) and the search becomes less sensitive. For this reason or the 
Table 6. Sensitivity depth $\mathcal{D}^{90 \%}$ from Eq. (2) corresponding to the $h_{0}^{90 \%}$ upper limits set by this search. The numbers in parenthesis are the sensitivity depths for the higher threshold search described in Ming et al. (2019).

\begin{tabular}{|c|c|c|c|}
\hline \multirow[b]{2}{*}{ Freq. range } & \multicolumn{3}{|c|}{ Sensitivity depth $\mathcal{D}^{90 \%}[1 / \sqrt{\mathrm{Hz}}]$} \\
\hline & J232327 & J085201 & $\mathrm{J} 1713$ \\
\hline $20-250 \mathrm{~Hz}$ & $65.4(60.3)$ & $84.2(76.5)$ & $89.4(82.9)$ \\
\hline $250-520 \mathrm{~Hz}$ & $58.0(54.5)$ & $74.3(70.1)$ & $85.6(79.1)$ \\
\hline $520-1500 \mathrm{~Hz}$ & $57.8(53.7)$ & $73.9(70.0)$ & $81.4(76.4)$ \\
\hline
\end{tabular}

same target, the sensitivity depth is higher at lower frequencies.

The upper limits on the amplitude can be readily translated in upper limits on the equatorial deformation $\epsilon$ of the star (assuming the moment of inertia in the direction of the spin axis $10^{38} \mathrm{~kg} \mathrm{~m}^{2}$ ) and r-mode amplitude $\alpha$ (under the same assumptions as Owen (2010)), as follows:

$$
\begin{aligned}
\varepsilon & =2.8 \times 10^{-7}\left(\frac{h_{0}}{3 \times 10^{-25}}\right)\left(\frac{D}{1 \mathrm{kpc}}\right)\left(\frac{1000 \mathrm{~Hz}}{f}\right)^{2} \\
\alpha & =8.4 \times 10^{-6}\left(\frac{h_{0}}{3 \times 10^{-25}}\right)\left(\frac{D}{1 \mathrm{kpc}}\right)\left(\frac{1000 \mathrm{~Hz}}{f}\right)^{3} .
\end{aligned}
$$

For the upper limits on $\varepsilon$ and $\alpha$ shown in Figure 4 we set the distances of the targets as follows: for J232327 we take $3.4 \mathrm{kpc}$, following the estimate $3.4_{-0.1}^{+0.3} \mathrm{kpc}$ of Reed et al. (1995); for J1713 we take $1.3 \mathrm{kpc}$ which is the center of the interval $1.3 \pm 0.4 \mathrm{kpc}$ of Cassam-Chenai et al. (2004); there is no consensus on the distance of J085201 so, from Allen et al. (2015) we consider two extremes: a distance of $0.2 \mathrm{kpc}$ and of $1 \mathrm{kpc}$, corresponding to the nearest and farthest that the object could be.

\section{CONCLUSIONS}

We follow-up about 10,000 sub-threshold candidates from an Einstein@Home search on O1 LIGO data for signals from J085201, J232327, and J1713 - the central compact objects in the supernova remnants Vela Jr., Cassiopeia A and SNR G347.3-0.5, respectively. At the threshold values set in this search a confident detection could not be claimed based on $\mathrm{O} 1$ data alone, but our follow-up searches on the newly released LIGO O2 data would be able to confirm a candidate stemming from a real signal.

Only one candidate from the first $\mathrm{O} 2$ follow-up warrants further investigation. This is at a frequency of about $369 \mathrm{~Hz}$ and comes from the search that targets emission from J1713. The follow-up search results on the remainder of $\mathrm{O} 2$ data $(\mathrm{O} 2 \mathrm{~b})$ fall short of the expected value and overall are not completely conclusive. If this candidate were of astrophysical origin it is unlikely that it would be a perfectly continuous and phase-coherent signal across all the observing periods that we have examined. We note that the search by Millhouse et al. (2020), which is robust against timing noise, does not produce any outlier consistent with ours, albeit the sensitivity of that search is not clear.

The timing solutions of Table 4 for the J1713 outlier imply a characteristic spin-down age of $\sim 1,300$ years, a spin-down energy loss rate of $1.6 \times 10^{40} \mathrm{erg} / \mathrm{s}$ and a surface magnetic field of $6 \times 10^{11} \mathrm{G}$. The characteristic age is consistent with that of a young NS associated with a undispersed supernova remnant $\left(\lesssim\right.$ few $\left.\times 10^{4}\right)$. The implied magnetic field is higher than of the detected central compact object pulsars but at the lower range for the rotation-powered pulsars. The large spin-down energy is in excess of the most energetic pulsars known (c.f. Crab pulsar, $\dot{E}=4.6 \times 10^{38} \mathrm{ergs} / \mathrm{s}$ ) and nearly all pulsars with $\dot{E} \gtrsim 4 \times 10^{36} \mathrm{ergs} / \mathrm{s}$ display bright X-ray and/or radio pulsar wind nebulae (Gotthelf 2004), with several notable exceptions. No wind nebula are associated with the known central compact object pulsars.

In the spirit of "leaving no stone unturned" we conduct an exhaustive timing search to identify an electromagnetic counterpart for this candidate. Since this source is only seen in X-ray emission, we analyze all available archival X-ray data on J1713 acquired with sufficient timing resolution. Although we do not find a significant signal at any of the expected frequencies, the sensitivity of the search is low, due to the many years gap between the LIGO and X-ray observations. This gap produces a very high number of search trials that results in a loss of sensitivity of our search.

New X-ray observations, contemporaneous with the LIGO runs, are planned, which should provide a more sensitive assessment ${ }^{3}$ of the J1713 candidate. A gravitational wave search in $\mathrm{O} 3$ data based on the parameters provided here should be easily able to shed light on the nature of this outlier. Noise investigations on the LIGO instruments could also reveal that the origin of outlier is a coherent contamination.

We set new upper limits on the intrinsic continuous gravitational wave amplitude from J232327, J085201, and J1713, improving on those already set in Ming et al. (2019). Our upper limits are below the age-based in-

\footnotetext{
${ }^{3}$ At the same X-ray signal strength the observation will be orders of magnitude more significant.
} 
direct upper limit (see for instance section 3 of Wette et al. (2008) for a definition) over most of the searched frequency range. Ellipticities at and above $10^{-6}$ are excluded for all targets above $1000 \mathrm{~Hz}$ (rotation frequency $500 \mathrm{~Hz}$ ) and for J1713 above $\sim 370 \mathrm{~Hz}$ (rotation frequency $185 \mathrm{~Hz}$ ). In fact for J1713 our results exclude ellipticities larger than $10^{-7}$ above $920 \mathrm{~Hz}$. This range of ellipticity values is interesting: neutron star crusts should readily be able to sustain ellipticities of the order of $10^{-6}$ (Johnson-McDaniel \& Owen 2013; Baiko \& Chugunov 2018). If r-modes are the gravitationalwave emission mechanism, our upper limits exclude amplitudes greater than $10^{-3}$ for all targets above $\sim 580$ $\mathrm{Hz}$ ( $\approx 435 \mathrm{~Hz}$ rotation frequency) and for J1713 above $\sim 360 \mathrm{~Hz}$ (rotation frequency $\approx 270 \mathrm{~Hz}$ ). In fact for J1713 our results exclude r-mode amplitudes larger than $5 \times 10^{-4}$ above $440 \mathrm{~Hz}$ (rotation frequency $\approx 330 \mathrm{~Hz}$ ). Estimates of the r-mode amplitude yield values of $10^{-3}$ or higher for several neutron-star hydrodynamic evolution scenarios (Bondarescu et al. 2009; Owen 2010). These are well within the reach of this search.

The focus of the work presented here is however not in the upper limits but in detection, i.e. investigating whether any of the $\approx 10,000$ outliers examined was due to a gravitational wave signal. Since the sensitivity of our search remains unmatched (Lindblom \& Owen 2020; Millhouse et al. 2020), investigating 10,000 new candidates further moves in uncharted territory.

This work also presents the first extensive follow-up of a continuous gravitational wave candidate in electromagnetic data and sets the stage for more investigations of this nature, including electromagnetic sub-threshold surveys followed by focused gravitational wave searches. It will be a combination of sensitive data and ingenuous search techniques, perhaps comprising multi-messenger observations, that will eventually secure the first detection of a continuous gravitational wave signal.

\section{ACKNOWLEDGMENTS}

The authors gratefully acknowledge the support of the many thousands of Einstein@Home volunteers, without whom this search would not have been possible.

M.A. Papa and B. Allen gratefully acknowledge support from NSF PHY grant 1816904. E.V. Gotthelf acknowledges support from NASA grant 80NSSC18K0452 \& SAO grant GO9-19046X.

The authors thank the LIGO Scientific Collaboration for access to the data and gratefully acknowledge the support of the United States National Science Foundation (NSF) for the construction and operation of the LIGO Laboratory and Advanced LIGO as well as the Science and Technology Facilities Council (STFC) of the United Kingdom, and the Max-PlanckSociety (MPS) for support of the construction of Advanced LIGO. Additional support for Advanced LIGO was provided by the Australian Research Council. This research has made use of data, software and/or web tools obtained from the LIGO Open Science Center (https://losc.ligo.org), a service of LIGO Laboratory, the LIGO Scientific Collaboration and the Virgo Collaboration, to which the authors have also contributed. LIGO is funded by the U.S. National Science Foundation. Virgo is funded by the French Centre National de Recherche Scientifique (CNRS), the Italian Istituto Nazionale della Fisica Nucleare (INFN) and the Dutch Nikhef, with contributions by Polish and Hungarian institutes. The follow-up studies for the J1713 outlier have also employed the PyFstat software suite (Ashton et al. 2020) and the Falcon search code (Dergachev \& Papa 2019).

\section{REFERENCES}

A. Singh, M. A. Papa, H. B. E., \& Walsh, S. 2017, Phys.

Rev. D, 96, 082003, doi: 10.1103/PhysRevD.96.082003

Aasi, J., et al. 2015a, Astrophys. J., 813, 39,

doi: 10.1088/0004-637X/813/1/39

—. 2015b, Class. Quant. Grav., 32, 074001,

doi: 10.1088/0264-9381/32/7/074001

Abbott, B., et al. 2020, Astrophys. J. Lett., 892, L3, doi: 10.3847/2041-8213/ab75f5

Abbott, B. P., et al. 2016, Phys. Rev. Lett., 116, 131103, doi: 10.1103/PhysRevLett.116.131103

—. 2017, Phys. Rev., D96, 122004, doi: 10.1103/PhysRevD.96.122004
-. 2019a, Phys. Rev., X9, 031040, doi: 10.1103/PhysRevX.9.031040

—. 2019b, Astrophys. J., 875, 122, doi: $10.3847 / 1538-4357 / \mathrm{ab} 113 \mathrm{~b}$

—. 2019c, Phys. Rev., D100, 024004, doi: 10.1103/PhysRevD.100.024004

AEI. 2020, Supplementary material for the publication, https://www.aei.mpg.de/continuouswaves/ O1GY-DirectedSearches

Allen, G., Chow, K., DeLaney, T., et al. 2015, Astrophys. J., 798, 82, doi: 10.1088/0004-637X/798/2/82 
Arvanitaki, A., Baryakhtar, M., \& Huang, X. 2015,

Physical Review D, 91, 084011

Ashton, G., Keitel, D., \& Prix, R. 2020, PyFstat-v1.3, 1.3, Zenodo, doi: 10.5281/zenodo.3620861. https://doi.org/10.5281/zenodo.3620861

Baiko, D., \& Chugunov, A. 2018, Mon. Not. Roy. Astron. Soc., 480, 5511, doi: 10.1093/mnras/sty2259

Behnke, B., Papa, M. A., \& Prix, R. 2015, Phys. Rev., D91, 064007, doi: 10.1103/PhysRevD.91.064007

Bondarescu, R., Teukolsky, S. A., \& Wasserman, I. 2009, Phys. Rev. D, 79, 104003, doi: 10.1103/PhysRevD.79.104003

Buccheri, R., et al. 1983, Astron. Astrophys., 128, 245

Cassam-Chenai, G., Decourchelle, A., Ballet, J., et al. 2004, Astron. Astrophys., 427, 199, doi: 10.1051/0004-6361:20041154

Cutler, C., \& Schutz, B. F. 2005, Phys. Rev., D72, 063006, doi: 10.1103/PhysRevD.72.063006

de Jager, O. C., Raubenheimer, B. C., \& Swanepoel, J. W. H. 1989, å, 221

Dergachev, V., \& Papa, M. A. 2019, Phys. Rev. Lett., 123, 101101, doi: 10.1103/PhysRevLett.123.101101

—. 2020, Phys. Rev. D, 101, 022001

Dreissigacker, C., Prix, R., \& Wette, K. 2018, Phys. Rev., D98, 084058, doi: 10.1103/PhysRevD.98.084058

Einstein@Home. 2019, The volunteer computing project Einstein@Home, https://einsteinathome.org/

Fesen, R. A., Hammell, M. C., Morse, J., et al. 2006, The Astrophysical Journal, 645, 283, doi: 10.1086/504254

Gotthelf, E. V. 2004, in IAU Symposium, Vol. 218, Young Neutron Stars and Their Environments, ed. F. Camilo \& B. M. Gaensler, 225

Gotthelf, E. V., Halpern, J. P., \& Alford, J. 2013, ApJ, 765

Horowitz, C. J., Papa, M. A., \& Reddy, S. 2020, Phys. Lett., B800, 135072, doi: 10.1016/j.physletb.2019.135072

Horowitz, C. J., \& Reddy, S. 2019, Phys. Rev. Lett., 122, 071102, doi: 10.1103/PhysRevLett.122.071102

Iyudin, A. 1999, Nucl. Phys. A, 654, 900c, doi: 10.1016/S0375-9474(00)88568-1

Jaranowski, P., Krolak, A., \& Schutz, B. F. 1998, Phys. Rev., D58, 063001, doi: 10.1103/PhysRevD.58.063001

Johnson-McDaniel, N., \& Owen, B. 2013, Phys. Rev. D, 88, 044004

Keitel, D. 2016, Phys. Rev., D93, 084024, doi: 10.1103/PhysRevD.93.084024

Keitel, D., Prix, R., Papa, M. A., Leaci, P., \& Siddiqi, M. 2014, Phys. Rev., D89, 064023, doi: 10.1103/PhysRevD.89.064023
LIGO. 2018, The O1 Data Release, https://www.gw-openscience.org/O1/, doi: https://doi.org/10.7935/K57P8W9D

—. 2019, The O2 Data Release, https://www.gw-openscience.org/O2/, doi: 10.7935/CA75-FM95

Lindblom, L., \& Owen, B. J. 2020, Phys. Rev. D, 101, 083023, doi: 10.1103/PhysRevD.101.083023

Mignani, R., Zaggia, S., De Luca, A., et al. 2008, Astron. Astrophys., 484, 457, doi: 10.1051/0004-6361:20079076

Millhouse, M., Strang, L., \& Melatos, A. 2020. https://arxiv.org/abs/2003.08588

Ming, J., Krishnan, B., Papa, M. A., Aulbert, C., \& Fehrmann, H. 2016, Phys. Rev., D93, 064011, doi: 10.1103/PhysRevD.93.064011

Ming, J., Papa, M. A., Krishnan, B., et al. 2018, Phys. Rev., D97, 024051, doi: 10.1103/PhysRevD.97.024051

Ming, J., Papa, M. A., Singh, A., et al. 2019, Phys. Rev., D100, 024063, doi: 10.1103/PhysRevD.100.024063

Nitz, A. H., Dent, T., Davies, G. S., et al. 2019, Astrophys. J., 891, 123, doi: 10.3847/1538-4357/ab733f

Owen, B. J. 2010, Phys. Rev., D82, 104002, doi: 10.1103/PhysRevD.82.104002

Owen, B. J., Lindblom, L., Cutler, C., et al. 1998, Phys. Rev., D58, 084020, doi: 10.1103/PhysRevD.58.084020

Palomba, C., et al. 2019, Phys. Rev. Lett., 123, 171101, doi: 10.1103/PhysRevLett.123.171101

Papa, M. A., et al. 2016, Phys. Rev., D94, 122006, doi: 10.1103/PhysRevD.94.122006

Pavlov, G. G., Sanwal, D., Kizlltan, B., \& Garmire, G. P. 2001, The Astrophysical Journal, 559, L131, doi: 10.1086/323975

Pferffermann, E., \& Aschenbach, B. 1996, in Proceeding of the International Conference on X-ray Astronomy and Astrophysics: Roentgenstrahlung from the Univers, $267-268$

Pletsch, H. J. 2008, Phys. Rev. D, 78, 102005. https://arxiv.org/abs/0807.1324

—. 2010, Phys. Rev. D, 82, 042002. https://arxiv.org/abs/1005.0395

Pletsch, H. J., \& Allen, B. 2009, Phys. Rev. Lett., 103, 181102

Prix, R. 2017, Coherent F-statistic on semi-coherent candidate $\mathrm{F}$. https://dcc.ligo.org/LIGO-T1700236/public

Reed, J. E., Hester, J., Fabian, A., \& Winkler, P. 1995, Astrophys. J., 440, 706, doi: 10.1086/175308

S. J. Zhu, M. A. P., \& Walsh, S. 2017, Phys. Rev. D, 96, 124007, doi: 10.1103/PhysRevD.96.124007 
Singh, A. 2017, Phys. Rev., D95, 024022, doi: 10.1103/PhysRevD.95.024022

Slane, P., Hughes, J. P., Edgar, R. J., et al. 2001, Astrophys. J., 548, 814, doi: 10.1086/319033

Tananbaum, H. 1999, Cassiopeia A, http:

//www.cbat.eps.harvard.edu/iauc/07200/07246.html

V. Dergachev, M. A. Papa, B. S., \& Eggenstein, H. B. 2019, Phys. Rev. D, D99, 084048
Vallisneri, M., Kanner, J., Williams, R., Weinstein, A., \& Stephens, B. 2015, J. Phys. Conf. Ser., 610, 012021, doi: 10.1088/1742-6596/610/1/012021

Venumadhav, T., Zackay, B., Roulet, J., Dai, L., \& Zaldarriaga, M. 2020, Phys. Rev. D, 101, 083030, doi: 10.1103/PhysRevD.101.083030

Wang, Z. R., Qu, Q. Y., \& Chen, Y. 1997, A\&A, 318, L59

Wette, K., et al. 2008, Class. Quant. Grav., 25, 235011, doi: 10.1088/0264-9381/25/23/235011

Zhu, S. J., Baryakhtar, M., Papa, M. A., et al. 2020. https://arxiv.org/abs/2003.03359

Zhu, S. J., et al. 2016, Phys. Rev., D94, 082008, doi: 10.1103/PhysRevD.94.082008 

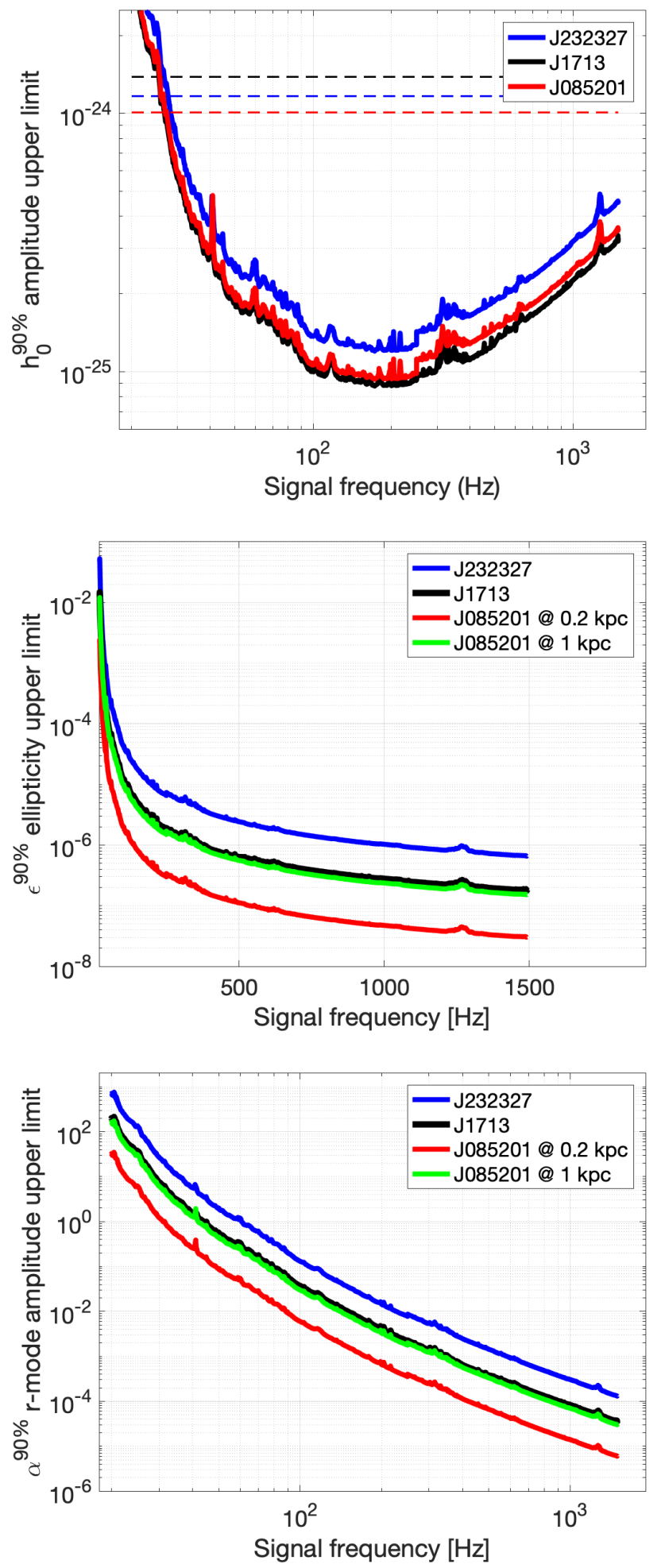

Figure 4. 90\% confidence upper limits on the gravitational wave amplitude, ellipticity and r-mode amplitude of continuous gravitational wave signals for the three targets. We assume J085201 is either at $0.2 \mathrm{kpc}$ or at $1 \mathrm{kpc}$, reflecting the large uncertainty on the actual distance of this object; for J232327 we assume a distance of $3.4 \mathrm{kpc}$ and for J1713 a distance of $1.3 \mathrm{kpc}$ (see text). The dashed lines in the top panel represent the age-based upper limits for the three targets. For the J085201 line we have assumed a distance of 0.2 kpc. 